\title{
Turbulent flow and loading on a tidal stream turbine by LES and RANS
}

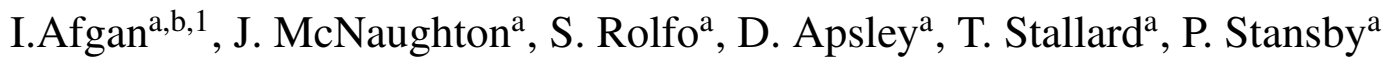 \\ ${ }^{a}$ Modelling $\mathcal{E}$ Simulation Centre, School of MACE, University of Manchester, M13 9PL, UK \\ ${ }^{b}$ Institute of Avionics $\mathcal{E}$ Aeronautics, Air University, E-9, Islamabad, Pakistan
}

\begin{abstract}
This paper presents results from numerical simulations of a 3-bladed horizontal axis tidal stream turbine. Initially, Reynolds Averaged Navier Stokes (RANS) k- $\omega$ Shear Stress Transport eddy-viscosity and Launder-Reece-Rodi models were used for code validation and testing of a newly implemented sliding mesh technique for an unstructured finite volume code. Wall- and blade-resolved large-eddy simulations (LES) were then performed to study the complete geometry at various tip speed ratios (TSR). Thrust and power coefficients were compared to published experimental measurements obtained from a towing tank for a range of $\operatorname{TSR}(4,5,6,7,8,9 \& 10)$ at a fixed hub pitch angle. A strong meandering is observed downstream of the supporting tower due to interaction between the detached tip vortices and vortex shedding from the support structure. The wake profiles and rate of recovery of velocity deficit show high sensitivity to the upstream turbulence intensities. However, the mean thrust and power coefficients were found to be less sensitive to the upstream turbulence. Comparisons between RANS and LES are also presented for the mean sectional blade pressures and mean wake velocity profiles. The paper also presents an overview of modelling and numerical issues relating to simulations for such rotating geometries.
\end{abstract}

(c) 2011 Published by Elsevier Ltd.

Keywords: LES, RANS, sliding meshes, tidal stream turbines.

\footnotetext{
${ }^{1}$ Corresponding Author: imran.afgan@manchester.ac.uk
} 


\section{Introduction}

With the world supply of oil and gas fast depleting there is increasing interest and investment in the renewable energy sector. One such major source is the untapped energy coming from oceans in the form of tidal or wave energy. Systems for generating useful electricity from this vast energy resource are in development, typically comprising arrays or farms of tidal stream turbines (TST). Over the last decade, the design of tidal stream devices has progressed rapidly and several horizontal-axis turbines are now undergoing prototype trials at offshore test sites. However, there is limited understanding of how the loading, power output and wakes of TST are affected by the ambient turbulence of the tidal flows that are suitable for energy extraction. Full scale experimental or field measurements of such devices and their wakes are both costly and extremely difficult, particularly when considering the operating environment and the extent of reliable data required. An alternative to field measurements is computational fluid dynamics (CFD), which can give a detailed insight into the flow physics, thereby enabling design optimization that can lead to low-cost yet high-energy efficient devices. This paper aims to present a detailed CFD investigation via Reynolds Averaged Navier Stokes (RANS) and wall-resolved large-eddy simulations (LES) to understand the flow physics and predict the behaviour of such a device under different operating conditions. To date there exists a shortage of experimental data for horizontal axis TST. Only a few laboratory scale experimental measurements exist. One set of experimental measurements comes from Bahaj et al. [1] and Bahaj et al. [2], which provide the mean power and thrust measurements at different immersion heights and hub pitch angles in both a cavitation tunnel and a towing tank. Later, Bahaj et al. [3] and Batten et al. [4] report on the development and verification of simulation tools based on blade element momentum theory.

On the numerical side Jimenez et al. [5] performed an LES of a wind turbine geometry with the actual turbine replaced by an approximate model of a concentrated drag force to study the wake behaviour. Calaf et al. [6] performed a suite of LES in which wake of arrays of turbines were modelled using the classical actuator disk concept. Churchfield et al. [7] also used a rotating actuator line method with LES to study wake propagation and power production for an array of TST. Concise comparisons are reported by Ferrer \& Munduate [8] between the conventional Blade Element Momentum (BEM) based methods and CFD (RANS) for wind turbines. Larsen et al. [9] developed a stochastic wake meandering model based on quasi-steady wake deficit and tip vortex instability models for turbines. Lu and PortéAgel [10] integrated a three-dimensional large-eddy simulation with an actuator line technique. More recently, Malki et al. [12] used a coupled blade element momentum method with RANS k- $\epsilon$ model for the performance evaluation of the laboratory scale turbine of Bahaj et al. [1]. Such numerical techniques are computational quite cheap and can be very useful for preliminary design and data analysis. However, they cannot be relied upon for a complete turbine performance analysis because of their inherent inability to resolve any transient flow features such as blade 
tip vortices, laminar to turbulent transition, flow separation and even downstream turbulence generation. On the other hand, McSherry et al. [11] numerically simulated the same laboratory scale turbine of Bahaj et al. [1] by resolving the complete blade geometry. The authors used the standard k- $\omega$ Shear Stress Transport (SST) model with a commercial solver to predict the mean blade loadings within 5 to $10 \%$ of the measurements. However, little or no wake data was reported.

The current study utilizes k- $\omega$ SST (Menter [13]) model, Launder-Reece-Rodi (LRR) (Launder et al. [14]) model and a fully wall- and blade-resolved LES of an identical TST geometry for a range of tip speed ratios. Both the transient and the mean rotor loadings are reported along with mean non-dimensional pressure coefficient $\left(\bar{C}_{P r}\right)$ for blade sections. The later part of the study focuses on effects of upstream turbulence on the turbine performance and wake recovery, where concise comparisons are given between RANS and LES at different operating conditions. An initial set of results for the simulations have already been presented in the $7^{\text {th }}$ International Symposium on Turbulence, Heat and Mass Transfer (see Afgan et al. [15]).

\section{Test Case Description}

A complete horizontal axis laboratory scale TST (comprising of 3 blades, nacelle and a mast) has been numerically simulated with both RANS and LES. The TST blade is constructed from seventeen NACA 6-series profiles with chord, thickness and pitch angles, all varying with the radial distance. All geometrical and model details conform to the experimental setup of Bahaj et al. [1]. The modelled domain is shown in figure 1a. In the experimental setup two different tip immersion cases were investigated with a varying pitch angle. For the current numerical study, the comparisons are based on the optimal pitch angle of $20^{\circ}$ for the deep tip immersion case only, where the rotor blade tip is approximately half rotor diameter below the water surface $(\mathrm{H}=0.55 \mathrm{D}$ case, in Bahaj et al. [1]). For the numerical setup a single turbine blade and its surrounding area is modelled in a $120^{\circ}$ segment, called the blade region. This blade region is then copied twice around the axis of the turbine, completing the inner cylindrical domain to rotate. The outer domain is made up of four different regions. The mast region includes the support structure of the TST model. The width (4.62D, span-wise $Y$ direction) and depth $(2.25 D, Z$ direction) of the domain match that in Bahaj et al. [1]. The domain length (stream-wise $X$ direction) is $3 D$ upstream of the turbine and $10 D$ downstream, where $D$ is the blade diameter.

The flow Reynolds number $\left(R e_{D, U_{0}}\right)$ based on inlet velocity $\left(U_{0}\right)$ and blade diameter $(D)$ is $1.2 \times 10^{6}$. The sides, base and top surfaces are set to symmetry conditions to model the experimental work described in Bahaj et al. [1] 
where a towing tank is used. The tip speed ratio (TSR), thrust and power coefficients are defined respectively as

$$
T S R=\frac{\Omega R}{U_{0}}
$$

$$
\begin{aligned}
C_{T} & =\frac{F_{X}}{0.5 \rho U_{0}^{2} A} \\
C_{P} & =\frac{T_{Q} \Omega}{0.5 \rho U_{0}^{3} A}
\end{aligned}
$$

where $\Omega$ is the rotation rate in $\mathrm{rad} / \mathrm{s}, \rho$ is the desinty of the fluid, $R$ is the blade radius $(m), A$ the projected rotor area $\left(m^{2}\right), F_{X}$ the thrust force $(N)$ and $T_{Q}$ the torque $(\mathrm{Nm})$. As the experimental domain is quite constrained, the blockage effects are likely to be significant and hence cannot be ignored. The blockage correction accounts for lower resultant velocities at the rotor in the absence of flow blockages, as if the turbine was being tested in an infinite domain. This blockage correction, given by Bahaj et al. [1], was applied for all parameters to obtain unconstrained actual conditions.

The current cases are simulated at different operating conditions with the TSR varying from 4 to 10 . For the first part of the study $1 \%$ upstream turbulence was chosen for RANS cases and a uniform inlet for LES. Later, simulations were performed for the optimal design $T S R$ of 6 with different levels of upstream turbulence.

\section{Numerical Method \& Grid Sensitivity Study}

The numerical simulations are performed using the Électricité de France $(E D F)$ open source CFD solverCode_Saturne (www.code-saturne.org). Code_Saturne is an unstructured collocated finite volume solver for incompressible flows (see Archambeau et al. [16] for solver details). For LES the filtered Navier-Stokes equations are written as

$$
\frac{\partial \widetilde{u}_{i}}{\partial x_{i}}=0
$$

$$
\frac{\partial \widetilde{u}_{i}}{\partial t}+\frac{\partial \widetilde{u}_{i} \widetilde{u}_{j}}{\partial x_{j}}=-\frac{1}{\rho} \frac{\partial \widetilde{p}}{\partial x_{i}}+v \frac{\partial^{2} \widetilde{u}_{i}}{\partial x_{j} \partial x_{j}}-\frac{\partial \tau_{\mathrm{ij}}}{\partial x_{j}}
$$

where $\widetilde{u}$ stands for the filtered velocity. In the above filtered Navier-Stokes equations the sub-grid scale tensor $\tau_{i j}$ needs to be modelled. The dynamic Smagorinsky model based on Germano et al. [17] and Lilly [18] minimization is used for the current procedure where the deviatoric part of the sub-grid scale tensor is given by :

$$
\tau_{i j}-\frac{1}{3} \tau_{k k} \delta_{i j}=-2 v_{t} \widetilde{S}_{i j}=-2\left(C_{S} \widetilde{\Delta}\right)^{2}\|S\| \widetilde{S}_{i j}
$$


where $\|S\|=\sqrt{2 \widetilde{S}_{i j} \widetilde{S}_{i j}}, \widetilde{S}_{i j}$ is the filtered strain rate tensor, $v_{t}$ the sub-grid scale viscosity, $\widetilde{\Delta}$ the filter width, $\delta_{i j}$ the Kronecker delta and $C_{S}$ the dynamic Smagorinsky constant. A fully hexahedral mesh is used with the filter width $\widetilde{\Delta}$ defined as $2 V^{\frac{1}{3}}$, where $V$ is the volume of a computational cell. An implicit (grid) filter is assumed whereas an explicit filter (based only on the immediate neighbours) is applied to compute the dynamic constant $C_{s}$. The dynamic constant $C_{s}$ is capped between values of 0 and 0.12 ( 0.24 when considering the global constant value i.e. $\left.2 \times C_{S}\right)$. The momentum equations are solved by considering an explicit mass flux estimated using Adams-Bashforth interpolation (the three components of the velocity are uncoupled). When a non-orthogonal grid is used, the matrix contains only the orthogonal contributions of the different operators; the non-orthogonal part is added to the right hand side of the transport equation.

For LES a $2^{\text {nd }}$ order centered scheme in space and time is used; Crank-Nicolson in time with a linearized convection and the $2^{\text {nd }}$ order Adams-Bashforth for the part of the diffusion involving the transposed gradient operator, which couples the velocity components. The code has been validated on several academic and industrial test cases; see Benhamadouche \& Laurence [19] and Afgan et al. [20].

A multi-block structured mesh was generated, where the blade section uses a C-mesh around the profile shape with the tip divided into several blocks to allow for a block-structured mesh. Each block was individually meshed with the use of non-conforming nodes to reduce the cell count away from the blades. A complete mesh sensitivity study was performed with different mesh resolutions (see table 1). The fine mesh (F1) was then chosen and a further refinement study was performed on it to study the effect of blade and outer mesh resolution on the mean power and thrust coefficients. Finally, based on the mesh convergence, the CF mesh was chosen for all fine LES simulations which has a total of 7.38 million control volumes. The final four meshes (reported in table 2), showed an error of less than 3\% for the mean power and thrust when compared to the experimental results. It should be noted here that for the final LES mesh (CF), the grid (spatial) scales were found to be substantially smaller than the Taylor micro-scales, thereby satisfying the LES mesh resolution requirement. This was estimated by computing dissipation and turbulent kinetic energy along various locations in the vicinity of the rotor for a very fine mesh ( 24 million cells). The numerical statistics such as mean and $r m s$ of the dynamic constant $C_{S}$ were also computed along with the contribution from the sub-grid scale model (not reported herein, see Afgan et al. [20] for similar details). This was found to be less than $5 \%$ in the vicinity of the turbine. For RANS three different mesh resolutions were also tested and a grid convergence study for thrust and power was performed to obtain the final mesh with 3.7 million cells (see table 3 for details). For RANS simulations, the scalable wall function of Grotjans \& Menter [21] was used, which ensures that the cell-centers of the control-volumes at the walls always lie within the buffer layer, thereby satisfying the inherent wall-function requirement. 
An isometric view of the complete TST (blades, nacelle and tower) along with an image of the full structured surface mesh are shown in figures 1a and $\mathrm{b}$, respectively. The local non-dimensional wall normal distribution $Y^{+}$for the same mesh at $T S R=6$ is shown in figure 1c. It can be observed that almost $99 \%$ of the blade surface exhibits a $Y^{+}$of less than 5, even at the blade tip. Similarly for the chosen LES fine mesh, the non-dimensional distances $\Delta X^{+}$ and $\Delta Z^{+}$near the blade are within the range of $5-10$. However, no attempt was made to resolve fully the far wake of the turbine as the focus of the current study was on turbine performance and effect of upstream turbulence. At each time-step the inner block rotates an amount $\Omega \Delta t$, where $\Delta t$ is the time step. The interface between the inner and outer blocks is controlled by a sliding-mesh method that was implemented within Code_Saturne as part of this work (see McNaughton et al. [22] for details). For each cell on the interface $(P)$ and its face $\left(F_{P}\right)$, a Dirichlet value is prescribed which is calculated as the average between the cell-center value and the value at a halo-point, $H$ :

$$
\phi_{F_{P}}=\frac{1}{2}\left(\phi_{P}+\phi_{H}\right)
$$

where $\phi$ is the flow value under consideration. The halo point is constructed by reflecting $P$ through $F_{P}$ as shown in figure $1 \mathrm{~d}$. The nearest cell-center to the halo-point is located at say point $E$. The value at $E$ is then extrapolated to the halo-point to find the value at the halo-point:

$$
\phi_{H}=\phi_{E}+\left.\frac{\partial \phi}{\partial \vec{x}}\right|_{E} \overrightarrow{E H}
$$

In the case of non-orthogonal meshes, the cell-center is projected onto this line and the value is found by extrapolation using the above equation. This sliding mesh procedure is semi-implicit in the pressure-velocity coupling algorithm making the coupling tight and maintaining the internal code convergence. This has been benchmarked on stationary and rotating cylinder test-cases (not reported herein). To improve the CPU time and scalability, an optimization for the communications is also carried out for the newly implemented sliding mesh technique as shown in figure 2. As a first step an improved performance is achieved by reordering some subroutines and the message passing interface (MPI) calls; this improves the general problem scalability (Version 1.1). To further improve performance, modifications to the parallel calls are made. This can best be described by the example of figure $2 \mathrm{a}$, where the left and right sides of a sliding-mesh interface are shown. The numbers in each cell represent their respective process numbers. For separate processes to communicate, an MPI call has to be made. In this example there are 8 processes numbered 0-7, although processes 6 and 7 are not part of the sliding-mesh interface.

The first developments (Version 1 and 1.1) use the MPI_Allgatherv call. Both these versions send and receive data between all processes as shown in figure $2 \mathrm{~b}$. By creating an additional MPI group this communication need only 
occur between processes involved on the sliding-mesh interface as shown in figure $2 \mathrm{c}$ (Version 2). The final result is a substantial reduction in computational time as shown by figure 2e, which shows the scalability of a 3.2 million mesh on a local cluster. For a further improvement, the processes on the left side need only communicate with the right, and vice-versa, as this is the only data they require. This reduces all communications even further as shown by figure 2d. However, this is not trivial, the MPI_Allgatherv function is a blocking call, which ensures that all the processes exchange data safely before the program can continue. To improve the communication scheme, two non-blocking calls are required: MPI_Isend and MPI_Irecv along with a wait function. This has been implemented as Version 2.2 with its performance clear again on the performance graph (see figure 2e).

It is also noted that processes need only talk to those immediately adjacent, for example process 0 only needs to send and receive data with process 4 and 5 in figure $2 \mathrm{a}$. This would reduce the communication time significantly. However, it requires not only a methodology to identify the adjacent processes but also an indication whether they need to communicate in the future or not. This latter idea has not yet been implemented, but will be incorporated for improving scalability for future large parallel calculations. As seen from figure 2e, subsequent developments have improved performances significantly and this is also confirmed by the scalability test performed on Cray XE6 HECToR (High End Computing Tera-scale Resource) for a much bigger mesh (results not shown in this paper). All LES simulations were performed with the initial development i.e. Version 1, whereas the RANS simulations are performed on a local cluster with the final development of Version 2.2. This new implementation of the sliding mesh has been validated and results are identical as improvements in the communication scheme only concerns operations on integers, leading to an increase in the speedup and an improvement in the scalability of the procedure.

\section{Results \& Discussion}

All LES simulations were performed on the Électricité de France (EDF) IBM Blue Gene P supercomputer using 2048 processors. The local Courant-Friedrichs-Lewy number (CFL) and the cell based Peclet number constrain the maximum time step that can be used for the wall-resolved LES. Thus, a substantial simulation time is required for a complete flow through pass (about 0.5 million time steps per case with a $\Delta t$ of $2 \times 10^{-5} s$ ). This corresponds to about 24 blade rotations for the optimal $T S R=6$ case. This is equivalent to about 0.14 Million CPU hours for each RANS simulation and 4.4 Million CPU hours for each LES. One can see the exponential increase in cost of the current (wall- and geometry-resolved) procedure as opposed to an actuator disc method used by Malki et al. [12] which required only 30 CPU hours for the same geometry. The sliding mesh procedure implemented in the current code requires very small time steps which is constrained by both the mesh rotation rate and the number of cells at the sliding interface. Tests conducted on a rotating cylinder (not reported in this paper) showed that the mesh rotation rate 
per iteration should be less than half the interface cell size. However, for the current LES the time step dictated by the wall-resolution at the trailing edge of the blade is substantially smaller than this requirement (about 10-30 times smaller)

\subsection{Steady uniform inlet flow}

A range of $\operatorname{TSR}$ (4 to 10) was simulated with both RANS k- $\omega$ SST model and LES with a uniform inlet. For all simulations, the flow was allowed to develop for at least 8-10 blade rotations and all mean statistics presented in this and the following sections are time averaged over at least the last 12 rotations. The variations of the blockage corrected mean thrust $\left(\bar{C}_{T}\right)$ and power $\left(\bar{C}_{P}\right)$ coefficients as a function of different TSR are shown in figure 3 . The comparisons are shown against measurements of Bahaj et al. [1] and numerical predictions of McSherry et al. [11]. It is observed from figure 3 that the predicted mean thrust $\left(\bar{C}_{T}\right)$ by [11] is within $8-10 \%$ of experiments. On the other hand, the mean power $\left(\bar{C}_{P}\right)$ predictions by [11] compare slightly better to the experiments (within 5\%). Mean thrust $\left(\bar{C}_{T}\right)$ and power $\left(\bar{C}_{P}\right)$ predictions from the current LES and RANS are always within 3\% of the experiments which is less than the scatter of the experimental data. The only exception is the $T S R=5$, where both the RANS models under predict the mean power $\left(\bar{C}_{P}\right)$ by about $10 \%$.

Figure 4 shows the mean pressure coefficient $\left(\bar{C}_{p r}\right)$ comparisons between RANS k- $\omega$ SST and LES at different $r / R$ span locations at $T S R=5,6$ and 8 , where $r$ is the normal distance along the blade span from the root chord. The pressure coefficient $\left(C_{p r}\right)$ is normalized based on the upstream inlet pressure $\left(p_{0}\right)$ and the local approach dynamic pressure; computed as $C_{p r}=\frac{p-p_{0}}{0.5 \rho\left(U_{0}^{2}+\Omega^{2} r^{2}\right)}$. It is observed from these figures that as the TSR increases, the difference between the suction and the pressure sides becomes smaller, leading to relatively small net lift and torque. It is further noted that the most affected region remains near the root chord, which shows considerable loss of lift as the TSR increases above the design conditions. The k- $\omega$ SST model also shows a similar trend. However, compared to LES, the difference between the suction and the pressure side from RANS simulations is slightly under-predicted regardless of the operating condition (TSR), thus leading to slightly lower predictions of the power coefficients. Interestingly, at design conditions $(T S R \approx 6)$ the $\mathrm{k}-\omega \mathrm{SST}$ model predictions are reasonably close to LES. However, at off-design conditions (TSR $=5$ ), the suction side pressure by both RANS models is under-predicted. This is indicative of local separation prediction by the RANS model which is not observed by LES.

Figure 5 shows the comparisons for mean pressure coefficient $\left(\bar{C}_{p r s}=\frac{p-p_{0}}{p_{S t a g}-p_{0}}\right)$, based on chord-wise stagnation pressure at $T S R=6$. This definition given by Ferrer \& Munduate [8] for the pressure coefficient, directly relates the pressure curve integral to the sectional normal force coefficient, thus showing the effective relative sectional loading. Looking at figure 5, it is now evident that with RANS k- $\omega$ SST model, the predictions near the tip of the blade show an under-prediction of the suction side pressure even at the design operating condition (see the dip/delayed RANS 
pressure profiles for tip locations $r / R=0.75$ and 0.87 in figure 5a). This is consistent with the slight under-prediction of the power coefficient by the RANS k- $\omega$ SST model, which is essentially derived from the pressure curve integral on the blade surface.

Comparisons between RANS and LES for time evolution of raw thrust $\left(C_{T}\right)$ and power $\left(C_{P}\right)$ coefficients for three blade rotations at TSR 6 are shown in figure 6. It is observed that the local frequency of oscillations of RANS is substantially lower than the LES. The instantaneous torque coefficients $\left(C_{q_{i}}\right)$ for the blades, about the non-rotational axes are shown in figure $7 \mathrm{a}$, where $C_{q_{i}}=T_{q_{i}} /\left(0.5 \rho U_{0}^{2} \pi R^{3}\right)$ with the index $i$ representing the non-rotational $(Y$ or $Z)$ axis about which the torque is reported. The local peaks in these plots represent the blade passing the mast; an effect which was not discernible from the instantaneous thrust coefficient plots due to local flow fluctuations. It is noted that the net torque along the non-rotational axis is quite small but not exactly zero. The net non-zero negative torque about the $Y$-axis is a direct result of the mast interference with the upstream flow, which causes a net loss of flow momentum on the upper side of the turbine compared to the lower side. On the other hand, the net non-zero torque about the $Z$-axis is due to the oblique flow onto the tower because of the rotation of the blades. The power spectrum density analysis (PSD) about these non-rotational axes are then shown in figure $7 \mathrm{~b}$, where one observes three times the blade rotational frequency ( $3 f$, where $f=\Omega / 2 \pi$ ) and its higher harmonics. The $-5 / 3$ slope of the PSD depicts the slope of the energy spectrum for homogeneous turbulence. This indicates that the implicit (grid) filter for LES lies within the inertial sub-range; thereby, showing the suitability of the mesh resolution.

Comparisons between the k- $\omega$ SST model and LES for the near blade-wake $(X / D=0.4)$ mean velocity profiles (normalized by $\Omega R$ ) are shown in figure 8 . In figure $8 \mathrm{a}$, along the vertical $Z$-axis $($ at $Y / D=0$ ), there is a difference in the mean velocity plateau between the top and bottom side of the nacelle (between $Z / D$ positive and negative locations). This mean plateau difference comes from the downstream mast which offers a blockage to the oncoming flow. With an increase in the $T S R$, the difference in the velocity profiles becomes smaller. The LES velocity profiles at the same location, shows a much flatter distribution compared to the k- $\omega$ SST model which exhibits a somewhat "W" shaped wavy profile. Close to $Z / D=0$, the sharp rise and dip in the velocity profile is due to the vortex shedding from the mast which is picked up by LES. This is in fact the strong helical wrapping of the flow around the nacelle which is visible only with the LES simulations (shown and discussed later in figure 11). The comparisons along the span $(Y$-axis) at various depths $(Z / D=0.25,0$ and -0.25$)$ are then shown in figures $8 \mathrm{~b}, \mathrm{c}$ and $\mathrm{d}$, respectively. It is observed that above the nacelle (at $Z / D=0.25$, figure $8 \mathrm{~b}$ ) and at mid-depth height $(Z / D=0$, figure $8 \mathrm{c})$, there still exists a lateral asymmetry in the mean velocity profile. This is indeed due to the net non-zero torque about the $\mathrm{Z}$-axis which is a direct result of the rotation of the blade causing an oblique flow onto the mast. Below the nacelle (at $Z / D=-0.25$, figure $8 \mathrm{~d}$ ), the mean velocity profiles are symmetric about the $Y / D=0$ location where both the 
k- $\omega$ SST model and the LES depict flat "U" shaped profiles. Admittedly, the RANS profiles are always slightly under predicted for all TSR.

Figure 9 shows the mean velocity profiles on the symmetry plane $(Y / D=0)$ at various downstream locations. In figure 9a which is just downstream of the mast, the plateau difference about $Z / D=0$ location once again shows the mast presence. Above the nacelle location (at $Z / D \approx 0.6$ ) there is a strong velocity peak, which is the effect of the tip vortex passing the mast. Both RANS and LES predict almost the same magnitude and shape for this velocity peak. Interestingly, at $Z / D=0$, the $\mathrm{k}-\omega \mathrm{SST}$ model predicts a sharp rise at $Z / D=0$ location, whereas LES shows a smooth "W" profile about this location. It is speculated that the RANS model is unable to predict accurately the shedding behind the tail cone of the nacelle. Indeed, close to the wake of any blunt object (inside the recirculation region), one would expect a "W" shaped velocity profile, which would eventually dissipate and change into a "V" or "U" shape moving downstream in the wake. This is what is observed in figures $9 \mathrm{~b}$ and c which are at $4 D$ and $9 D$ downstream of the turbine rotor, respectively. At $9 D$ downstream, the k- $\omega$ SST profile has fully recovered into a smooth "U" shape. Interestingly, the mast effect above the nacelle location $(Z / D$ positive side) is also no longer visible where both left and right side profiles are now almost symmetrical. However, with LES the asymmetry of the profile about the nacelle location is still visible even at $9 D$ downstream. Comparisons between the RANS k- $\omega$ SST model and the LES for the normalized mean turbulent kinetic energy $\left(\bar{k} / U_{0}^{2}\right)$ at $X=0.4 D$ downstream of the rotor plane are shown in figure 10 . For the RANS results, contributions from both the resolved and the modelled parts are presented. It can be observed that RANS predictions are always under predicted regardless of the location. In fact observing closely, it is noticed that above the nacelle at $Z / D=5$ (figure 10b), the RANS model does not pickup the higher energy content simply because of the inability to predict the tip vortices and the blockage effects of the downstream mast; one observes almost no difference between RANS results above or below the nacelle (see figures $10 \mathrm{~b} \& \mathrm{c}$ ). This is not the case with LES, which shows strong kinetic energy peaks about $Y / D= \pm 0.5$ above the nacelle (see figure $10 \mathrm{~b}$ ).

The iso-Q structures $\left(-0.5\left(\Omega_{i j} \Omega_{i j}-S_{i j} S_{i j}\right)\right)$ presented in figure 11a show the complexity of the underlying flow field. For computations, $Q$ is defined as $Q=-0.5\left(S_{i j} S_{i j}-\Omega_{i j} \Omega_{i j}\right)$, herein, normalized by $\left(Q=\alpha\left(U_{0} / d\right)^{2}\right)$ where $\alpha$ is a non-dimensional constant set in order to clearly visualize the flow structures (for the current cases $\alpha$ is chosen to return a value of $Q=1 / s^{2}$ ). In this figure, the evolution of the helical tip vortices can clearly be seen. Shedding can also be observed in the wake of the tail cone of the nacelle, the effect of which was earlier seen and reported in the mean wake velocity profiles. The vortex shedding from the vertical support structure (i.e. the mast) interferes strongly with these wing tip vortices, resulting in a complex flow meandering near the mast region. The mast itself shows a strong vertical 'roller' of vorticity which becomes inclined as it propagates downstream and is eventually broken down by the large scale structures of the tip vortices. On the surface of the nacelle one observes a strong three dimensional 
helical wrapping of the flow, which extends downstream even beyond the mast, thereby adding to the shedding of the TST structure's tail cone. The iso-surfaces of velocity, extracted around the blade tip region show the expansion of the helical tip vortices as it propagates downstream (see figure 11c).

Comparisons of instantaneous streamlines for k- $\omega$ SST model and LES at various sectional $Y Z$ planes in the blade wake for TSR 6 are presented in figure 12. The sectional planes in this figure cut through the helical tip vortices seen before. In figure $12 \mathrm{~b}$ which is at $X / D=0.156$ (immediate wake of the blade), these 3 strong tip vortices can be observed by both RANS and LES. As one moves downstream, these vortices expand in size and start moving outwards (see figure 12c). At $X / D=0.5$ (figure 12d), which is just upstream of the mast, $\mathrm{k}-\omega \mathrm{SST}$ does not predict the tip vortex structures anymore. However, in the same figure, LES still shows the 3 tip vortices along with shedding from the nacelle. The top two vortices by LES are somewhat distorted due to the presence of the mast. Further downstream of the mast in figure 12e \& f, the RANS k- $\omega$ SST model picks up some shedding from the vertical support structure. On the other hand LES shows strong shedding from both the nacelle and the mast. The lower tip vortex in its distorted form is also visible, whereas the other two vortices have now been completely destroyed.

\subsection{Effect of upstream turbulence}

The effect of various levels of upstream turbulence $(1 \%, 10 \%$ and $20 \%)$ were studied in detail by both RANS and LES for the optimal design condition (TSR 6). For RANS k- $\omega$ SST model, the mixing-length $l_{m}$ was set at 0.7 times the turbine immersion depth i.e. $0.84 \mathrm{~m}$ as suggested by Gant \& Stallard [23]. From this the turbulent kinetic energy $(k)$ and turbulent dissipation $(\varepsilon)$ were specified at the inlet as

$$
k=\frac{3}{2}(I U)^{2} \quad\{\text { where } U=1.5 \mathrm{~m} / \mathrm{s} \text { and } I=1 \%, 10 \% \& 20 \%\}
$$

$$
\varepsilon=\frac{C_{\mu}^{3 / 4} k^{3 / 2}}{l_{m}} \quad\left\{\text { where } l_{m}=0.5880\right\}
$$

where $I$ is the inlet turbulence intensity and $C_{\mu}$ is a constant (0.09). For LES, both the mean and fluctuating variables are required at the inlet for development of a realistic state of turbulence at a certain distance downstream. Conventional synthetic turbulence generation methods require a long time to develop, leading to large upstream domain lengths (see Tabor \& Baba-Ahmadi [24]).

A more effective way to introduce turbulent fluctuations at the inlet is to use the Synthetic Eddy Method (SEM) by Jarrin et al. [25]. The method is based on reconstruction of the turbulent fluctuations as a sum of all eddies inside an arbitrarily defined box, which contains the inlet surface. In case of non-isotropic turbulence, the method uses the Lound's coefficients to deform the eddy shape, thereby loosing the divergence free condition. A way to overcome 
this problem is to apply the SEM procedure to the vorticity field, and from it obtain the desired velocity field (see Poletto et al. [26]). This procedure, called Divergence Free Synthetic Eddy Method (DFSEM), tends to maintain the divergence free condition. However, from a numerical pint of view, a true divergence free condition can only be satisfied up to the machine precision and that too only in case of a solver with a staggered arrangement. For a collocated arrangement, such as the one under consideration, the DFSEM produces synthetic turbulence more similar to real turbulence. The DFSEM method not only requires a shorter upstream development length but also helps in reducing pressure oscillations at the inlet which are typical of the SEM methodology.

Comparisons between RANS and LES for inlet turbulence levels (10 \& 20\%), 1D upstream of the turbine rotor along the lateral $(Y)$ and vertical $(Z)$ axis are shown in figure 13 . The highly fluctuating turbulence intensity profiles are due to the methodology of DFSEM, where vortex structures of various size and intensities are convected downstream. The turbulence intensity wavelength for DFSEM is around $0.2-0.25 \mathrm{D}$, which is about $10 \%$ of the domain height (5\% when compared to the domain width); this is close to the Prandtl mixing length. It is also observed from this figure that the mean turbulence levels $1 D$ upstream of the rotor for both RANS and LES are within the comparison range, showing a small amount of decay as they are convected downstream. For RANS simulations, $10 \%$ and $20 \%$ inlet turbulence translates into $9.5 \%$ and $18.1 \%$, whereas for DFSEM these translate into $9.2 \%$ and $17.6 \%$, respectively.

A comprehensive comparison of all tested cases at different operating conditions is listed in table 4 . It is noted from this table that with the exception of $T S R=5$, the mean $C_{T}$ and $C_{P}$ for all tested RANS models and LES are within $3 \%$ of the experiments for the entire range of $\operatorname{TSR}$ (which is less than the scatter of the measurements). It is further noted that with an increase in the upstream turbulence levels, the mean blade loadings remain unaffected, although the turbulent length scale is almost equal to the blade diameter; showing a steady decrease of less than $0.5 \%$ for all cases. On the other hand the rms of fluctuations of thrust $\left(C_{T}^{\prime}\right)$ and power $\left(C_{P}^{\prime}\right)$, seemed to be slightly more sensitive, showing a gradual increase with increasing upstream inlet turbulence, see table 4 for details. Similar conclusions can be drawn when looking at the mean and rms pressure coefficients (not reported herein) with different upstream turbulence levels.

The effect of the changing upstream turbulence intensity on the wake of complete TST structure is shown by contour plots of mean velocity in the $Y Z$ plane, figure 14 . Here, a criterion of $95 \%$ of velocity is used to define the wake edge, which is depicted as a solid black contour line. It is observed that even though the statistics have been averaged long enough, the net imbalance effect of the momentum is seen in the near wake $(1 D)$ plots for all turbulence intensities. Furthermore, at the same location $1 D$ downstream, with increasing turbulence intensity, the wake becomes narrower. In figure $14 \mathrm{a}$ (1\% turbulence intensity case), it is also observed that the mast effect and net imbalance of momentum is somewhat still visible even for the $9 D$ downstream location. On the other hand, the 
mast effect is lost as one moves downstream from $1 D$ to $4 D$ for the $10 \%$ turbulence intensity case. However, the net imbalance can still be seen at $4 D$ in figure $14 \mathrm{~b}$, which is recovered fully at the $9 D$ downstream location. Finally, for the $20 \%$ turbulence intensity case, both the mast effect and momentum imbalance are recovered well before even $4 D$ location (see figure 14c). This favorable wake recovery with an increase in the upstream turbulence intensity can also be observed from the velocity deficit plots at various downstream locations in figure 15. It can thus be concluded from all these results that with increasing upstream turbulence intensity, the complete TST structure wake becomes narrower and its recovery seems to be faster (also evident from the instantaneous velocity particle tracers in the same figure). Indeed, this is very important for tidal stream turbine farms.

\section{Conclusions}

The current paper presents a new methodology for sliding meshes which is implemented in an open source CFD solver. Simulations are then performed utilizing this new formulation to predict mean and unsteady information about the wakes and power production for a wall- and blade-resolved laboratory-scale turbine. Within the current framework, the accuracy of the numerical simulations is assessed by direct comparison of mean loads to published experimental data. For tip speed ratios greater than 6 (up to 10), mean thrust and power coefficients are predicted to within $3 \%$ of the experimental data by both LES and RANS. For lower tip speed ratios similar accuracy is observed by LES but RANS under predicts the power coefficient by up to $10 \%$. Analysis of the chord variation of pressure coefficient indicates that this is because both RANS models under predict suction pressure, particularly near the leading edge. Analysis of the time-varying thrust and torque confirms that LES resolves unsteady loads at the blade passing frequencies and into the inertial sub-range.

LES yields detailed behavior for blade tip vortices and their interaction with the supporting tower, neither of which are captured completely by the RANS models. This is observed by flow visualization and from the radial variation of turbulent kinetic energy. At the design point $(T S R=6)$, turbulent kinetic energy across the width of the tip vortices immediately downstream $(X=0.4 D)$ differs by less than $20 \%$ between LES and RANS. However, RANS predicts lower turbulent kinetic energy in this region at all other operating points. Further downstream, the detached tip vortices interact with vortex shedding from the mast and a strong three-dimensional helical wrapping of the flow is observed around the nacelle and downstream. Interaction with the mast also causes a net imbalance of momentum about the lateral and vertical axes, leading to small non-zero moments.

At the optimum design condition, the effect of different upstream turbulence intensities is also investigated for the turbine performance and its wake. An increase in the upstream turbulence by small amounts decreases the wake recovery distance. This is indeed very important from the perspective of installing arrays of tidal turbines for mass 
56

power production. The mean thrust and power coefficients are not very sensitive to the upstream turbulence. The RANS predictions indicate an increase of the rms of the fluctuating thrust and torque with turbulence intensity. The LES predicts a higher fluctuating load than RANS but shows less variation with turbulence intensity. Although the computational cost of LES is far greater than RANS, the blade-resolved simulations presented show that this approach provides greater insight into flow-physics and unsteady loading, particularly for operation away from the design point. In the future studies, the authors plan to include wave motion on the free surface by simulating the tidal cycle-variation of the inflow. Numerical predictions of mean and unsteady loads will then be assessed against field measurements from a full-scale prototype turbine.

\section{Acknowledgments}

This research was performed as part of the Reliable Data Acquisition Platform for Tidal (ReDAPT) project commissioned and funded by the Energy Technologies Institute (ETI). The authors are highly grateful to EDF for additional funding and access to its High Performance Computing (HPC) facilities. The authors are also grateful to UK s9 Turbulence Consortium (UKTC) for providing additional computing time on UK National Supercomputing Service (HECToR). 


\section{References}

[1] A. S. Bahaj, W. M. J. Batten, A. F. Molland and J. R. Chaplin. Experimental Investigation into the Hydrodynamic Performance of Marine Current Turbines. Sustainable Energy Series Report, 3 (2005).

[2] A. S. Bahaj, A. F. Molland, J. R. Chaplin and W. M. J. Batten. Power and Thrust Measurements of Marine Current Turbines under various Hydrodynamic Flow Conditions in a Cavitation Tunnel and a Towing Tank. Renewable Energy, 32(3), 407-426 (2007).

[3] A. S. Bahaj, W. M. J. Batten and G. McCann. Experimental Verifications of Numerical Predictions for the Hydrodynamic Performace of Horizontal Axis Marine Current Turbines. Renewable Energy, 32(15), pp. 2479-2490 (2007).

[4] W. M. J. Batten, A. S. Bahaj, A. F. Molland and J. R. Chaplin. The Prediction of the Hydrodynamic Performance of Marine Current Turbines. Renewable Energy, 33(5), pp. 1085-1096 (2008).

[5] A. Jimenez, A. Crespo, E. Migoya and J. Garcia. Advances in Large-Eddy Simulation of a Wind Turbine Wake. In Journal of Physics: Conference Series, 75(1), 012041 (2007).

[6] M. Calaf, C. Meneveau and J. Meyers. Large Eddy Simulation Study of Fully Developed Wind-Turbine Array Boundary Layers. Physics of Fluids, 22, 015110 (2010).

[7] M. J. Churchfield, Y. Li and P. J. Moriarty. A Large-Eddy Simulation Study of Wake Propagation and Power Production in an Array of Tidal-Current Turbines. In Proc. 9th European Wave and Tidal Energy Conference, University of Southampton, UK, 2011.

[8] E. Ferrer and X. Munduate. Wind Turbine Blade Tip Comparison using CFD. J. Phys. Conf. Ser., 75, 012005 (2007).

[9] G. C. Larsen, H. A. Madsen, F. Bingöl, J. Mann, S. Ott, J. N. Sørensen, V. Okulov, N. Troldborg, M. Nielsen, K. Thomsen, T. J. Larsen and R. Mikkelsen. Dynamic Wake Meandering Modeling. In Ris $\phi$ National Laboratory report, Tec. Uni. Denmark. Risø-R-1607(EN), (2007).

[10] H. Lu and F. Porté-Agel. Large-Eddy Simulation of a Very Large Wind Farm in a Stable Atmospheric Boundary Layer. Phy. Fluids. 23, 065101 (2011).

[11] R. McSherry, J. Grimwade, I. Jones, S. Mathias, A. Wells and A. Mateus. 3D CFD Modelling of Tidal Turbine Performance with Validation against Laboratory Experiments. In Proc. 9th European Wave and Tidal Energy Conference, University of Southampton, UK, 2011.

[12] R. Malki, A. J. Williams, T. N. Croft, M. Togneri and I. Masters. A Coupled Blade Element Momentum-Computational Fluid Dynamics model for Evaluating Tidal Stream Turbine Performance. Applied Mathematical Modelling. 37, pp 3006-3020 (2013).

[13] F. R. Menter. Two-equation Eddy-Viscosity Turbulence Models for Engineering Applications. AIAA., Vol. 32, No. 8, pp 1598-1605 (1994).

[14] B. E. Launder, G. J. Reece and W. Rodi. Application of the Energy Dissipation Model of Turbulence to the Calculation of Flow near a Spinning Disc. Letters in Heat and Mass Transfer, 1(2), pp. 131-138 (1974).

[15] I. Afgan, J. McNaughton, D. Apsley, S. Rolfo, T. Stallard and P. Stansby. Large-Eddy Simulation of a 3-Bladed Horizontal Axis Tidal Stream Turbine: Comparisons to RANS and Experiments. In Proc. Turbulence, Heat and Mass Transfer 7, Sicily, Italy, (2012).

[16] F. Archambeau, N. Mechitoua and M. Sakiz. Code_Saturne: A Finite Volume Code for the Computation of Turbulent Incompressible Flows - Industrial Applications. Int. J. Finite Vol., 1(1), 2004.

[17] M. Germano, U. Piomelli, P. Moin and W. Cabot. A Dynamic Subgrid-scale Eddy Viscosity Model. Phys. Fluids. 3(7), pp. 1760-1765 (1991).

[18] D. Lilly. A Proposed Modification of the Germano Subgrid-Scale Closure Method. Phys. Fluids., 4, pp. 633-635 (1992).

[19] S. Benhamadouche and D. Laurence. LES, coarse LES, and Transient RANS Comparisons on the Flow Across Tube Bundles. Int. J. Heat and Fluid Flow. 24, pp. 470-479 (2003).

[20] I. Afgan, Y. Kahil, S. Benhamadouche and P. Sagaut. Large Eddy Simulation of the Flow Around Single and two Side-by-Side Cylinders at Subcritical Reynolds Numbers. Phys. Fluids. 23(7), 075101. (2011).

[21] H. Grotjan and F. R. Menter. Wall Functions for Industrial Applications. In Proceedings of Computational Fluid Dynamics, Chichester, UK, ECCOMAS. Vol. 1, No. 2, pp 1112-1117, (1998).

[22] J. McNaughton, I. Afgan, D. Apsley, S. Rolfo, T. Stallard and P. Stansby. A Robust Sliding-Mesh Interface Procedure and its Application to the CFD Simulation of a Tidal-Stream Turbine. Int. J. Numerical Methods in Fluids. Article under review (2012).

[23] S. Gant and T. Stallard. Modelling a Tidal Turbine in Unsteady Flow. Proceedings of the Eighteenth International Offshore and Polar Engineering Conference. Vancouver, Canada. (2008).

[24] G. Tabor and M. Baba-Ahmadi. Inlet Conditions for Large Eddy Simulation: A review. Computers $\mathcal{E}$ Fluids. 39, pp. 553567 (2010).

[25] N. Jarrin, R. Prosser, J. Uribe, S. Benhamadouche and D. Laurence. Reconstruction of Turbulent Fluctuations for Hybrid RANS/LES Simulations using a Synthetic-Eddy Method. Int. J. Heat and Fluid Flow. 30, pp. 435442 (2009).

[26] R. Poletto, A. Revell, T. Craft and N. Jarrin. Divergence Free Synthetic Eddy Method for Embedded LES Inflow Boundary Conditions. In Seventh International Symposium On Turbulence and Shear Flow Phenomena (TSFP-7), Ottawa, Canada, 2011. 
Table 1. Comparison of mean thrust $\left(\bar{C}_{T}\right)$ and power $\left(\bar{C}_{P}\right)$ coefficients at $T S R 6$ for mesh sensitivity study.

\begin{tabular}{c|c|c|c|c}
\hline Mesh & No. of Cells & $Y^{+}$ & $\overline{C_{T}}$ & $\overline{C_{P}}$ \\
\hline C1 & 1474290 & $30-150$ & 0.707 & 0.356 \\
C2 & 2741434 & $30-150$ & 0.737 & 0.376 \\
M1 & 3361544 & $30-150$ & 0.741 & 0.372 \\
M2 & 5104553 & $30-150$ & 0.747 & 0.379 \\
F1 & 7941749 & $3-15$ & 0.792 & 0.425 \\
F2 & 14210511 & $3-15$ & 0.782 & 0.421 \\
VF1 & 18003415 & $3-15$ & 0.788 & 0.431 \\
VF2 & 21334826 & $3-15$ & 0.794 & 0.427 \\
\hline
\end{tabular}


Table 2. Mesh details (cells in each region corresponding to figure 1a) and further grid sensitivity study for mean blade loadings at TSR 6 for LES.

\begin{tabular}{c|c|r|c|c|c|c|c|c|c|c}
\hline Mesh & Inner & \multicolumn{1}{|c|}{ Mast } & Outer & Upstrm. & Dnstrm. & Blade & Total & $Y^{+}$ & $\overline{C_{T}}$ & $\overline{C_{P}}$ \\
\hline CC & 1792092 & 863060 & 194480 & 130350 & 273448 & 1895028 & 5148458 & $3-15$ & 0.774 & 0.417 \\
CF & 2111892 & 1795974 & 449280 & 261500 & 791700 & 1895028 & 7305374 & $3-15$ & 0.781 & 0.431 \\
FC & 1792092 & 863060 & 194480 & 130350 & 273448 & 3255960 & 6509390 & $3-15$ & 0.792 & 0.439 \\
FF & 2111892 & 1795974 & 449280 & 261500 & 791700 & 3255960 & 8666306 & $3-15$ & 0.788 & 0.437 \\
\hline
\end{tabular}


Table 3. Mesh details and grid sensitivity study for mean blade loadings at $T S R$ for RANS.

\begin{tabular}{c|c|c|c|c|c}
\hline Mesh & No. cells turbine & No. cells domain & No. cells total & $\overline{C_{T}}$ & $\overline{C_{P}}$ \\
\hline Coarse & 823977 & 627356 & 1451333 & 0.8094 & 0.444 \\
Medium & 3078636 & 627356 & 3705992 & 0.827 & 0.463 \\
Fine & 5336976 & 627356 & 5964332 & 0.825 & 0.463 \\
\hline
\end{tabular}


Table 4. Comparison of corrected tip speed ratios $\left(T S R_{c}\right)$, mean thrust $\left(\bar{C}_{T}\right)$, mean power $\left(\bar{C}_{P}\right)$, various upstream inlet turbulence intensities (T.I.) and turbulence $1 D$ upstream of rotor (T.I.P), between k- $\omega$ SST, LLR, LES and experimental measurements (Bahaj et al. [1]).

\begin{tabular}{|c|c|c|c|c|c|c|c|}
\hline- & $T S R$ & (T.I./T.I.P) \% & $T S R_{C}$ & $\bar{C}_{T}$ & $C_{T}^{\prime}$ & $\bar{C}_{P}$ & $C_{P}^{\prime}$ \\
\hline \multirow{8}{*}{$k-\omega S S T$} & 5 & 1 & 4.89 & 0.659 & 0.00117 & 0.368 & 0.00152 \\
\hline & \multirow{3}{*}{6} & 1 & 5.82 & 0.780 & 0.00106 & 0.425 & 0.00100 \\
\hline & & $10 / 9.51$ & 5.83 & 0.775 & 0.00184 & 0.412 & 0.00102 \\
\hline & & $20 / 18.1$ & 5.82 & 0.776 & 0.00261 & 0.400 & 0.00121 \\
\hline & 7 & 1 & 6.74 & 0.852 & 0.00143 & 0.430 & 0.00150 \\
\hline & 8 & 1 & 7.66 & 0.890 & 0.00097 & 0.400 & 0.00072 \\
\hline & 9 & 1 & 8.57 & 0.915 & - & 0.345 & - \\
\hline & 10 & 1 & 9.48 & 0.937 & - & 0.271 & - \\
\hline \multirow{6}{*}{$L R R$} & 5 & 1 & 4.89 & 0.663 & - & 0.376 & - \\
\hline & 6 & 1 & 5.82 & 0.779 & - & 0.422 & - \\
\hline & 7 & 1 & 6.75 & 0.843 & - & 0.420 & - \\
\hline & 8 & 1 & 7.67 & 0.884 & - & 0.390 & - \\
\hline & 9 & 1 & 8.59 & 0.908 & - & 0.332 & - \\
\hline & 10 & 1 & 9.51 & 0.923 & - & 0.247 & - \\
\hline \multirow{7}{*}{ LES } & 4 & 1 & 3.95 & 0.586 & - & 0.356 & - \\
\hline & 5 & 1 & 4.88 & 0.709 & - & 0.416 & - \\
\hline & \multirow{3}{*}{6} & 1 & 5.82 & 0.781 & 0.00383 & 0.434 & 0.00197 \\
\hline & & $10 / 9.2$ & 5.82 & 0.780 & 0.00385 & 0.425 & 0.00232 \\
\hline & & $20 / 17.6$ & 5.82 & 0.779 & 0.00391 & 0.425 & 0.00235 \\
\hline & 7 & 1 & 6.74 & 0.840 & - & 0.430 & - \\
\hline & 8 & 1 & 7.68 & 0.885 & - & 0.403 & - \\
\hline \multirow{9}{*}{ Bahaj et al. [1] } & 5 & - & 4.88 & 0.695 & - & 0.426 & - \\
\hline & 6 & - & 5.67 & 0.776 & - & 0.445 & - \\
\hline & 7 & - & 6.49 & 0.850 & - & 0.454 & - \\
\hline & 7 & - & 6.56 & 0.828 & - & 0.421 & - \\
\hline & 7 & - & 6.90 & 0.865 & - & 0.421 & - \\
\hline & 7 & - & 7.09 & 0.881 & - & 0.438 & - \\
\hline & 8 & - & 8.24 & 0.931 & - & 0.390 & - \\
\hline & 9 & - & 8.74 & 0.954 & - & 0.352 & - \\
\hline & 10 & - & 9.71 & 0.958 & - & 0.294 & - \\
\hline
\end{tabular}


(a)

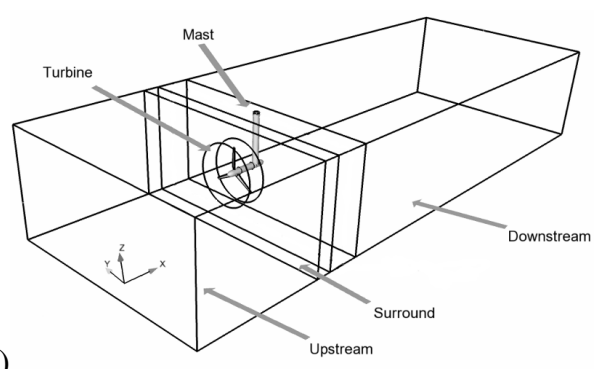

(c)

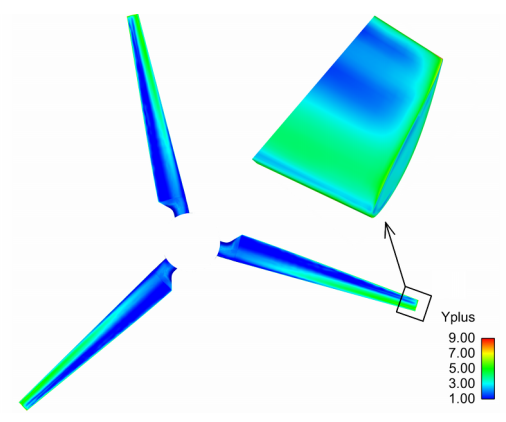

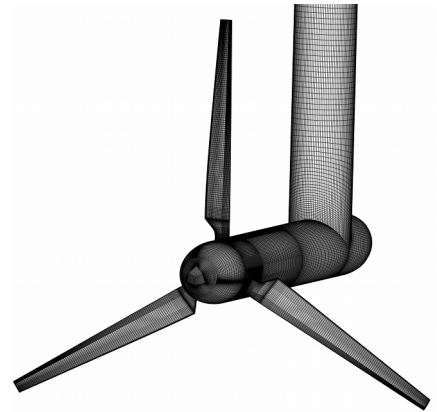

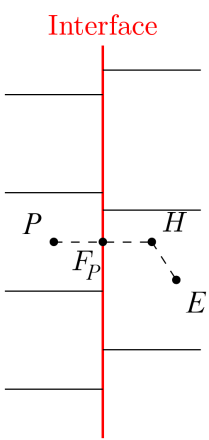

(b)

(d)

Figure 1. Geometry and mesh details (a) Full TST CFD domain (b) View of the surface mesh (c) Local $Y^{+}$distribution on the blade surface for $T S R 6$ (d) Interpolation of face value between host-cell and halo-point. 


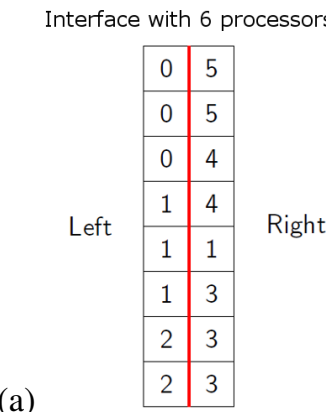

(b)
Number of communications 15

(c)

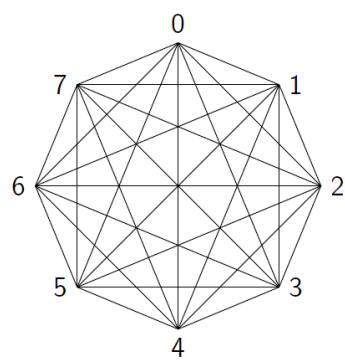

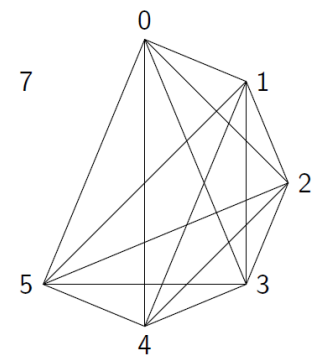

Number of communications 10

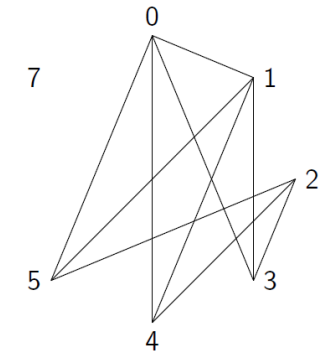

(d)

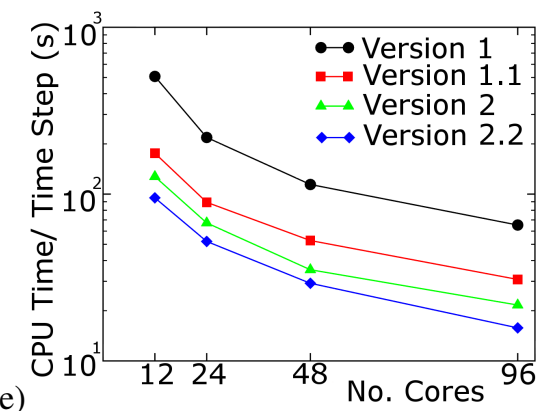

Figure 2. Sliding mesh procedure optimization (a) Example interface with 6 processors (b) Version 1 \& 1.1: Communications between all processors (c) Version $2 \&$ 2.2: Communication between neighbour processors (d) Version 3: Optimized communication between immediate neighbours only (e) Speedup with different versions on a 3.2 million mesh. 

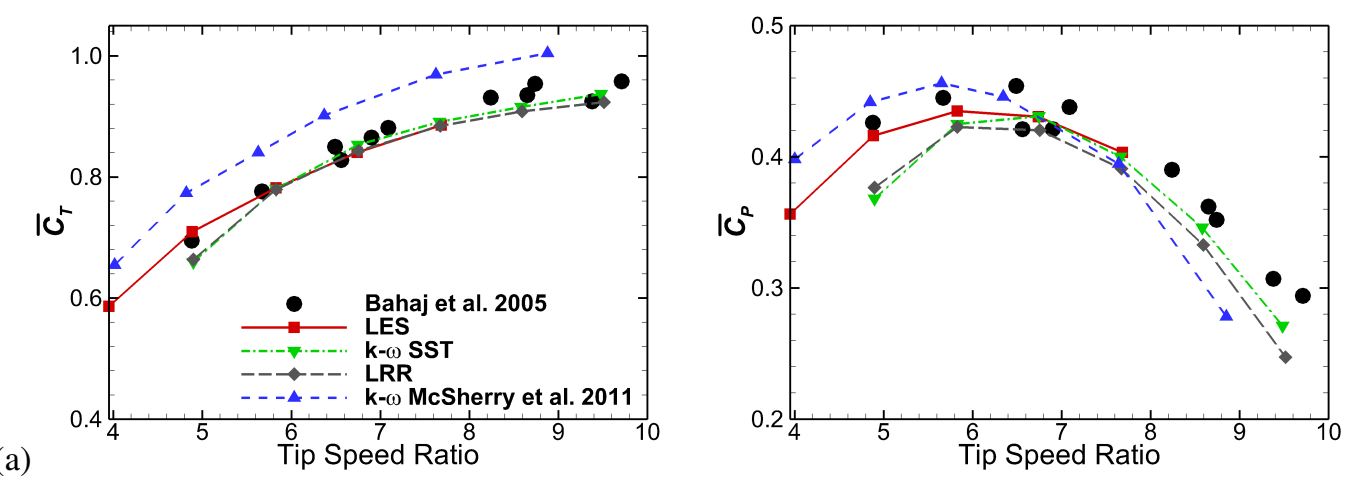

(b)

Figure 3. Comparison of blockage corrected mean coefficients at different TSR. (a) Mean $\bar{C}_{P}$ (b) Mean $\bar{C}_{T}$. 

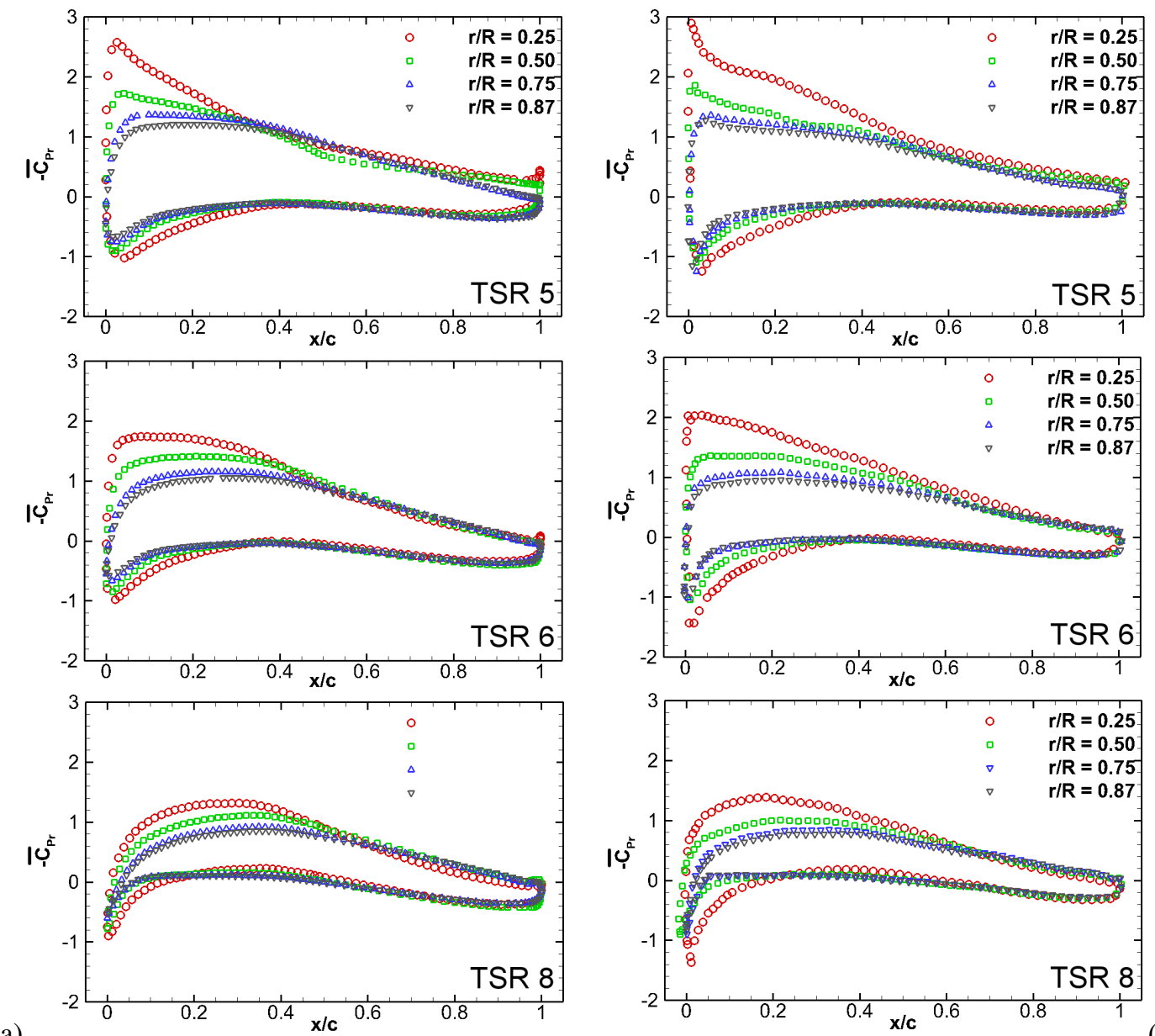

(a)

Figure 4. Mean pressure coefficient $\left[\bar{C}_{p r}=\left(p-p_{0}\right) /\left(0.5 \rho\left(U_{0}^{2}+\Omega^{2} r^{2}\right)\right)\right]$ at different $r / R$ span locations for $T S R$ 5, 6 and 8 . (a) RANS k- $\omega$ SST model (b) LES. 
(a)
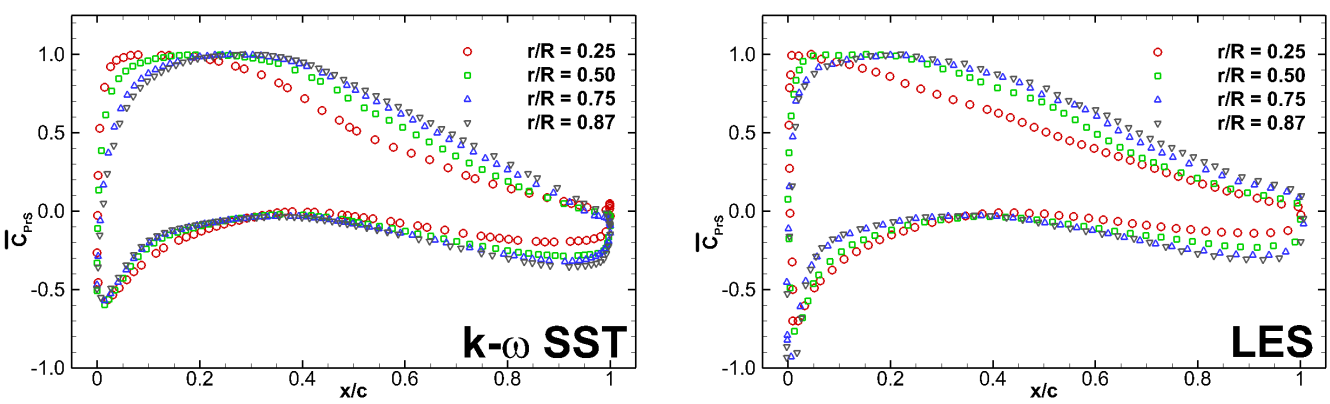

(b)

Figure 5. Mean pressure coefficient $\left[\bar{C}_{p r S}=\left(p-p_{0}\right) /\left(p_{S t a g}-p_{0}\right)\right]$ at different $r / R$ span locations for TSR 6. (a) RANS k- $\omega$ SST model (b) LES. 
(a)
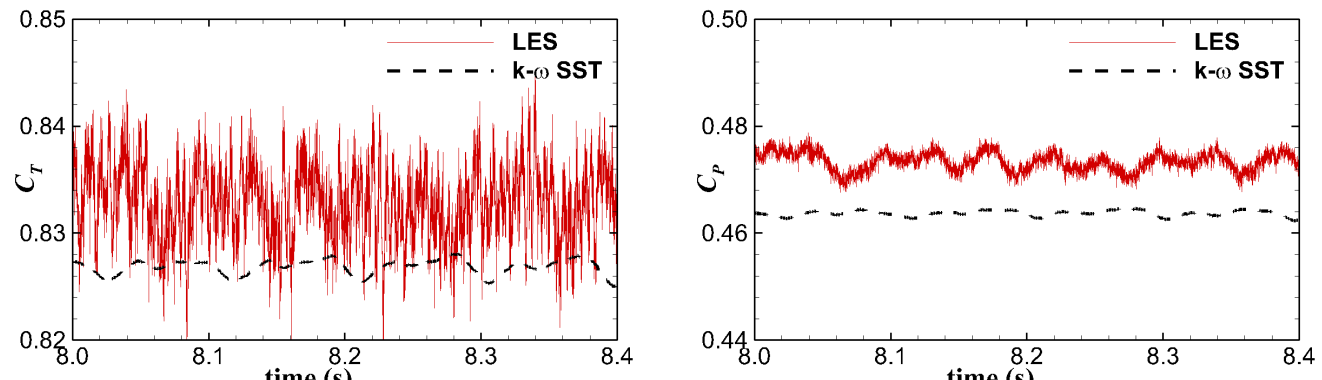

time (s)

Figure 6. Comparison of raw transient power and thrust coefficients at TSR 6 between LES and k- $\omega$ SST model for three blade rotations (a) Raw thrust coefficient $C_{T}=\left[F_{X} /\left(0.5 \rho U_{0}^{2} A\right)\right]$ (b) Raw power coefficient $C_{P}=\left[T_{Q} \Omega /\left(0.5 \rho U_{0}^{3} A\right)\right]$. 

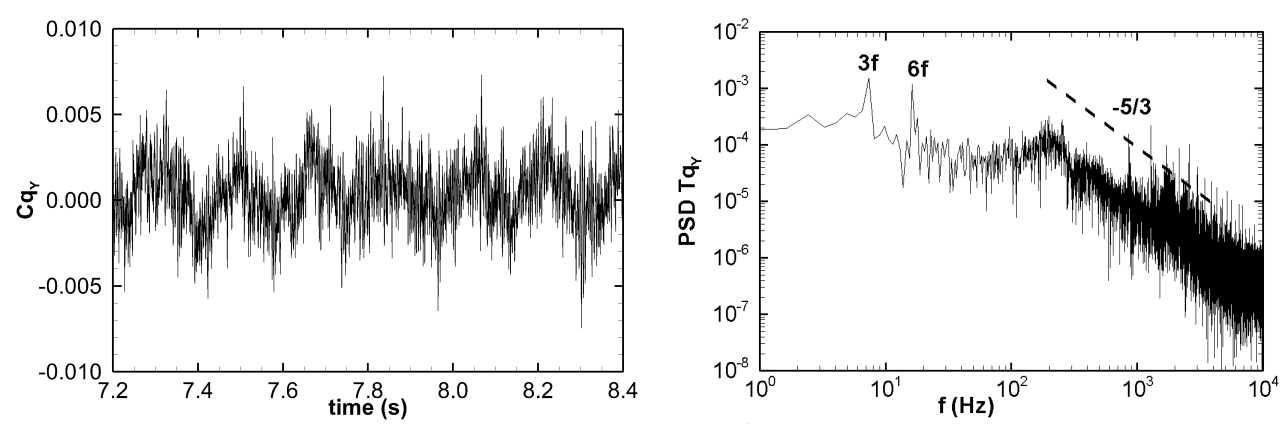

(a)
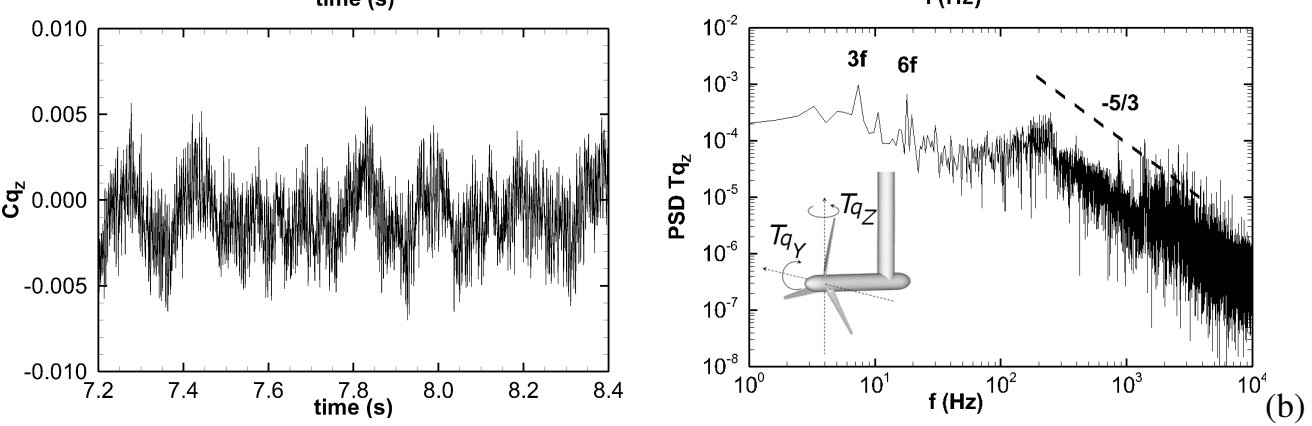

Figure 7. (a) Instantaneous torque coefficients along $Y\left(C_{q_{Y}}=T_{q_{Y}} /\left(0.5 \rho U_{0}^{2} \pi R^{3}\right)\right)$ and $Z\left(C_{q_{z}}=T_{q_{z}} /\left(0.5 \rho U_{0}^{2} \pi R^{3}\right)\right)$ axes (b) Power spectrum density of the instantaneous torques $\left(T_{q Y}, T_{q Z}\right)$ for LES at $T S R 6$. 
(a)
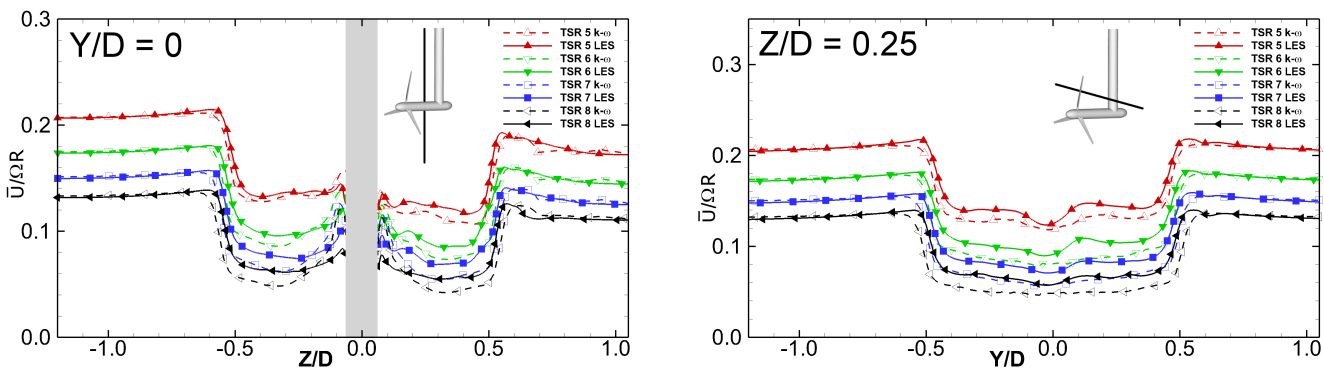

(c)
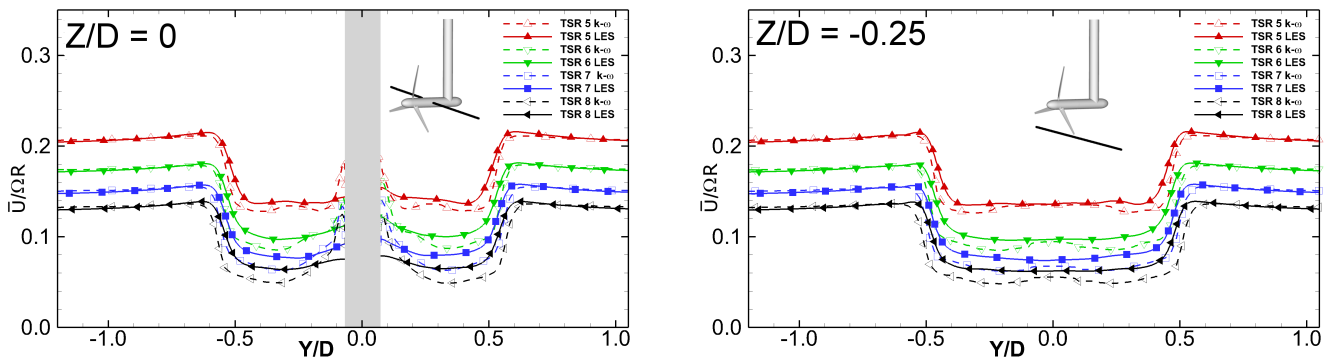

Figure 8. Mean velocity profiles in the immediate blade wake at $X / D=0.4$ for TSR 6 case (a) At $Y / D=0$ along vertical $Z$ axis (b) At $Z / D=0.25$ along span $Y$ axis (c) At $Z / D=0$ along span $Y$ axis (d) At $Z / D=-0.25$ along span $Y$ axis. 

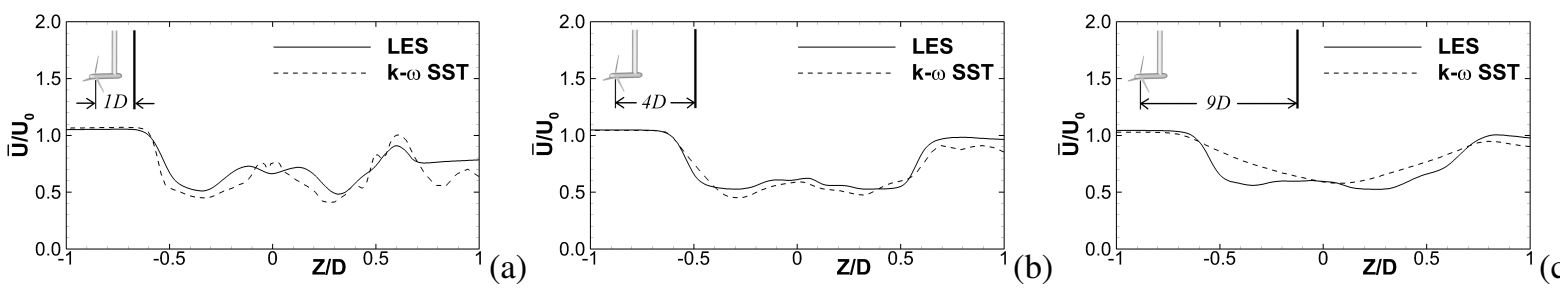

Figure 9. Mean velocity profile comparisons between k- $\omega$ SST model and LES at symmetry plane $(Y / D=0)$ at various downstream locations for TSR 6 with uniform inlet (a) $X=1 D$ (b) $X=4 D$ (c) $X=9 D$. 
(a)
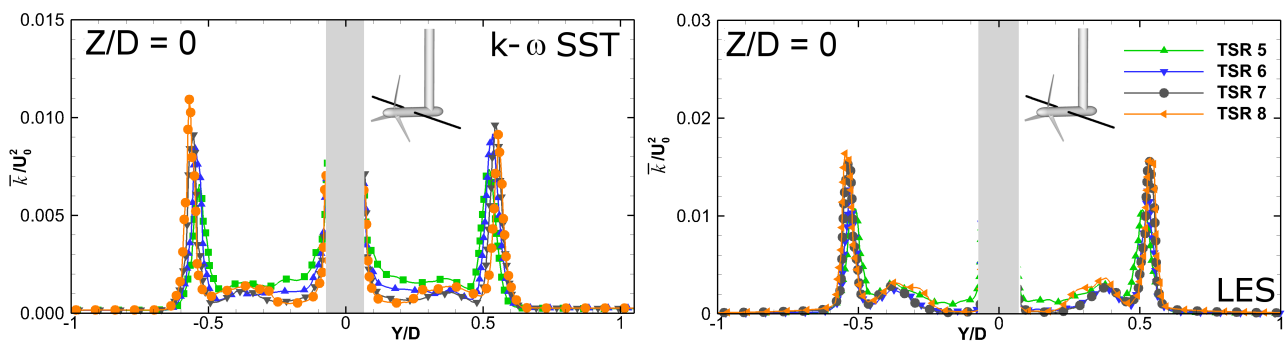

(b)
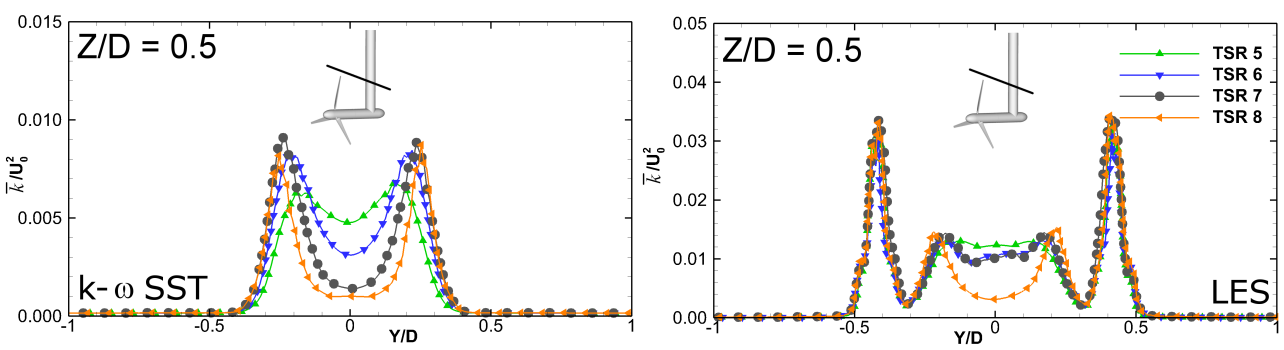

(c)
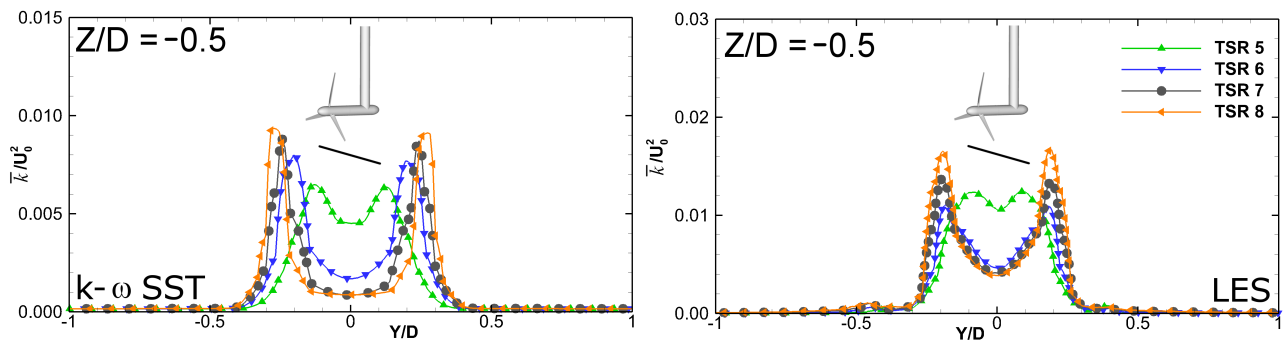

Figure 10. Mean kinetic energy profiles in the immediate blade wake at $X / D=0.4$ along span ( $Y$-axis) for $T S R 6$ case (a) $Z / D=0$ (b) $Z / D=0.5$ (c) $Z / D=-0.5$. 


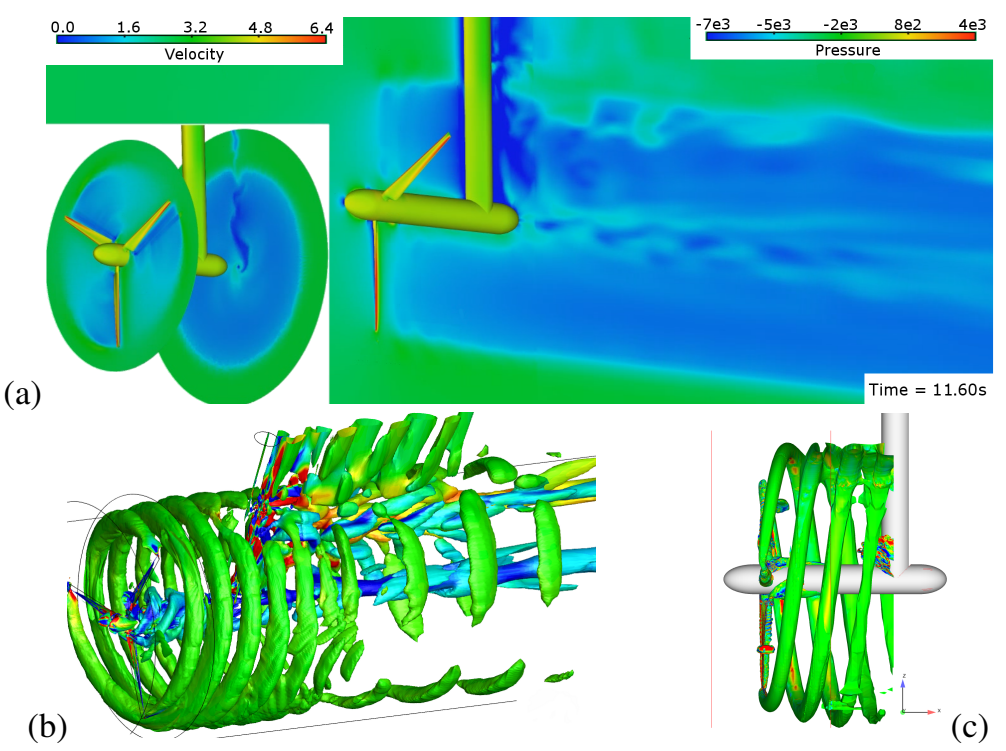

Figure 11. (a) Instantaneous velocity and pressure (on the body) contours at $T S R=6$ (b) Isometric view of the iso-Q $\left(-0.5\left(\Omega_{i j} \Omega_{i j}-S_{i j} S_{i j}\right)\right.$ ) coloured by mean vorticity along rotational axis (c) Iso-surfaces of velocity extracted around the blade tip region. 
(a)

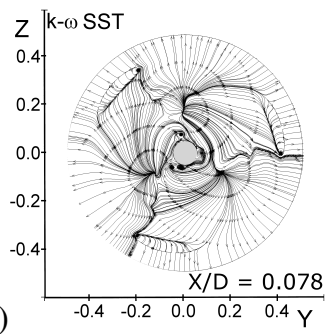

(c)
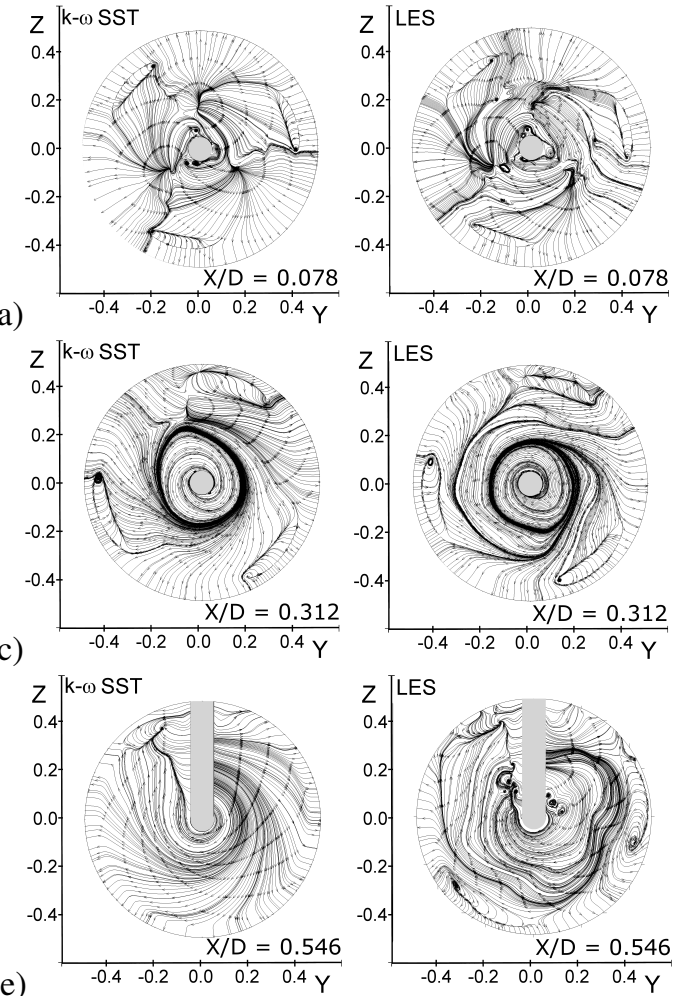

(b)
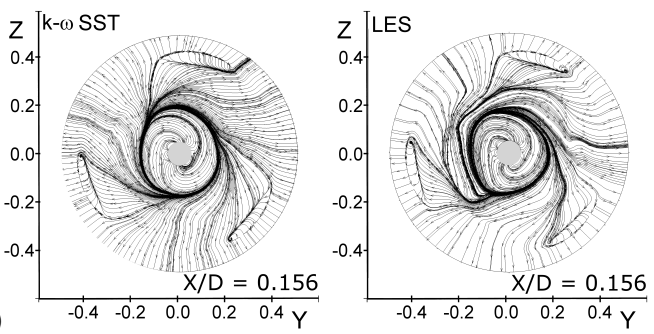

(d)
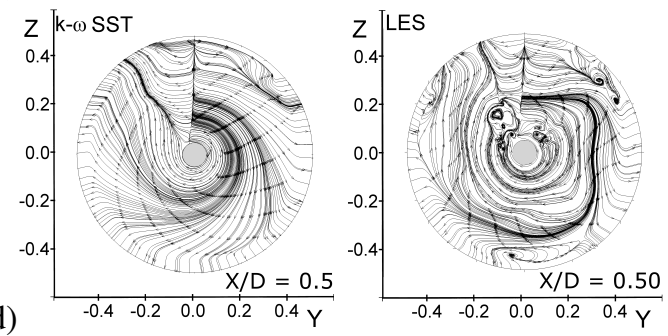

(f)
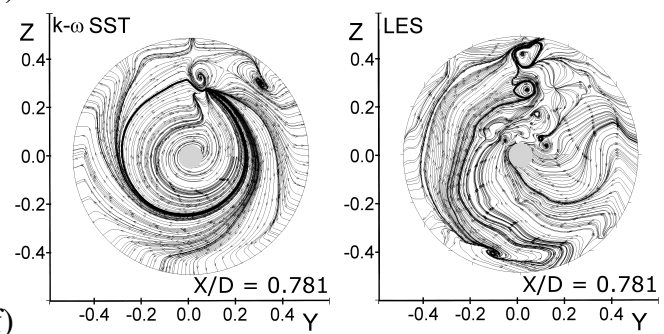

Figure 12. Comparisons of instantaneous velocity streamlines for k- $\omega$ SST model and LES at various sectional $Y Z$ planes in the blade wake for $T S R 6$ case at (a) $X / D=0.078$ (b) $X / D=0.156$ (c) $X / D=0.312$ (d) $X / D=0.50$ (e) $X / D=0.546$, (f) $X / D=0.781$. 

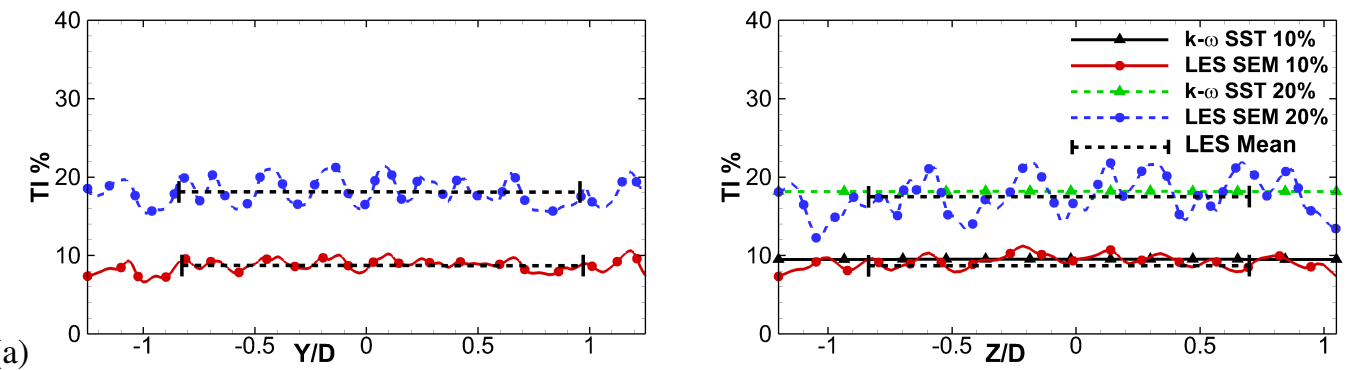

Figure 13. Comparison between RANS and LES DFSEM for different upstream turbulence intensity profiles (a) Variation along the span $Y$-axis (b) Variation along the depth $Z$-axis. 


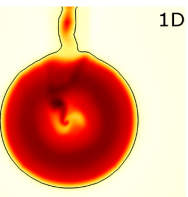

(a)

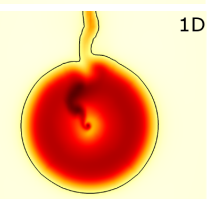

(b)

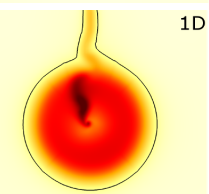

(c)

D

D
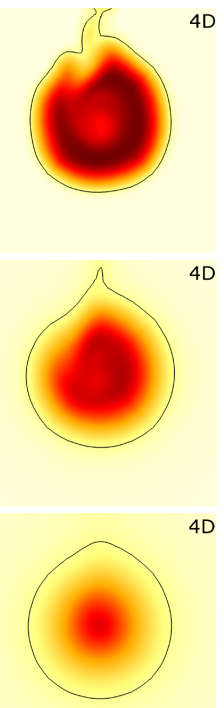

$4 \mathrm{D}$

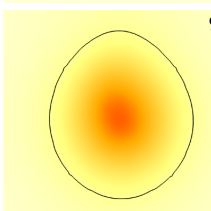

$9 \mathrm{D}$

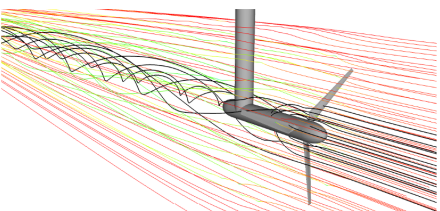

k- $\omega$ SST $1 \%$ TI

$9 \mathrm{D}$

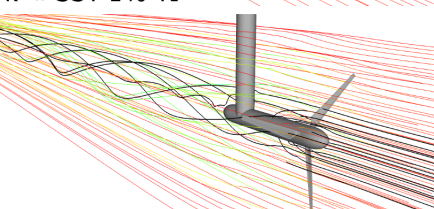

k- $\omega$ SST $10 \%$ TI

D

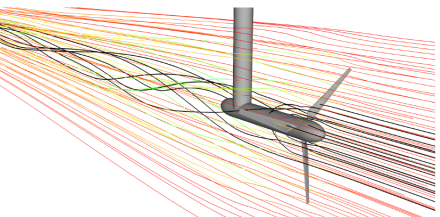

k- $\omega$ SST $20 \%$ TI

Figure 14. Instantaneous velocity particle tracers $(r . h . s)$ and mean velocity contours at various downstream locations with k- $\omega$ SST model at $T S R$ 6 (a) $1 \%$ Turbulence Intensity (TI) (b) $10 \%$ TI (c) $20 \%$ TI. 


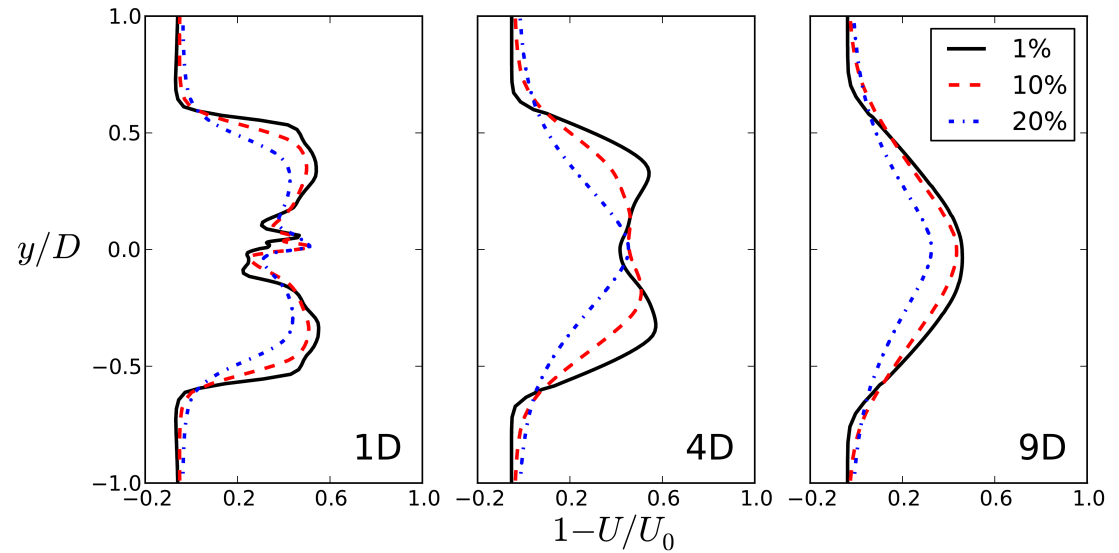

(a)

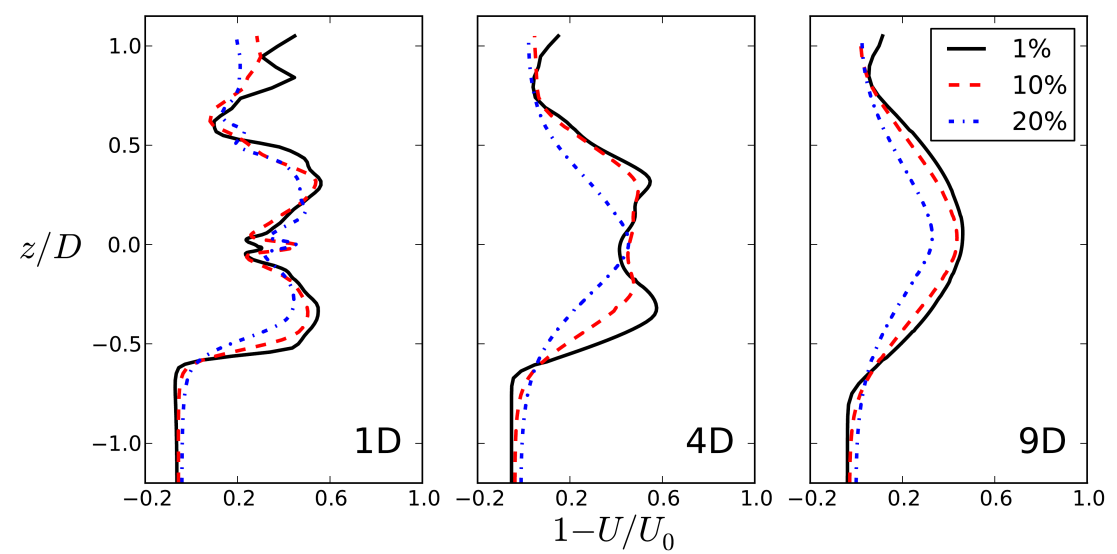

(b)

Figure 15. Velocity deficit comparison for different inlet turbulent intensities with k- $\omega$ SST model (TSR 6) at various downstream locations (a) Along span $Y$-axis (b) Along depth $Z$-axis. 
